\title{
Experimental characterization of the effects induced by passive plasma lens on high brightness electron bunches
}

Cite as: Appl. Phys. Lett. 111, 184101 (2017); https://doi.org/10.1063/1.4999010

Submitted: 04 August 2017 . Accepted: 10 October 2017 . Published Online: 30 October 2017

(D) A. Marocchino, M. P. Anania, (D) M. Bellaveglia, A. Biagioni, (D) S. Bini, F. Bisesto, E. Brentegani, (D) E. Chiadroni, A. Cianchi, M. Croia, D. Di Giovenale, M. Ferrario, (D) F. Filippi, A. Giribono, V. Lollo, (D) M. Marongiu, (D) A. Mostacci, G. Di Pirro, (D. Rompili, S. Romeo, (D) A. R. Rossi, J. Scifo, V. Shpakov, C. Vaccarezza, F. Villa, and A. Zigler

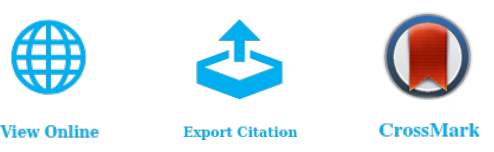

\section{ARTICLES YOU MAY BE INTERESTED IN}

Experimental characterization of active plasma lensing for electron beams Applied Physics Letters 110, 104101 (2017); https://doi.org/10.1063/1.4977894

Guiding of charged particle beams in curved capillary-discharge waveguides AIP Advances 8, 015326 (2018); https://doi.org/10.1063/1.5011964

Comparative study of active plasma lenses in high-quality electron accelerator transport lines Physics of Plasmas 25, 056702 (2018); https://doi.org/10.1063/1.5018001

\section{Meet the Next Generation of Quantum Analyzers And Join the Launch Event on November 17th}

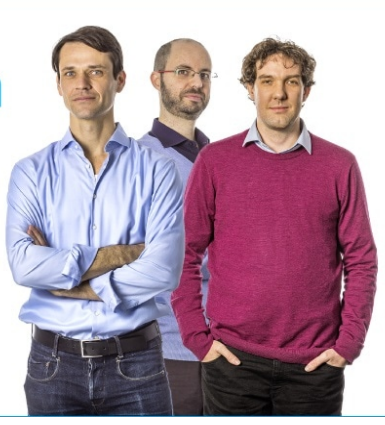

\section{Register now}

Zurich Instruments 


\title{
Experimental characterization of the effects induced by passive plasma lens on high brightness electron bunches
}

\author{
A. Marocchino, ${ }^{1, a)}$ M. P. Anania, ${ }^{1}$ M. Bellaveglia, ${ }^{1}$ A. Biagioni, ${ }^{1}$ S. Bini, ${ }^{1}$ F. Bisesto, ${ }^{1}$ \\ E. Brentegani, ${ }^{1}$ E. Chiadroni, ${ }^{1}$ A. Cianchi, ${ }^{2}$ M. Croia, ${ }^{1}$ D. Di Giovenale, ${ }^{1}$ M. Ferrario, ${ }^{1}$ \\ F. Filippi, ${ }^{3}$ A. Giribono, ${ }^{3}$ V. Lollo, ${ }^{3}$ M. Marongiu, ${ }^{3}$ A. Mostacci, ${ }^{3}$ G. Di Pirro, ${ }^{1}$ R. Pompili, ${ }^{1}$ \\ S. Romeo, ${ }^{1}$ A. R. Rossi, ${ }^{4}$ J. Scifo, ${ }^{1}$ V. Shpakov, ${ }^{1}$ C. Vaccarezza, ${ }^{1}$ F. Villa, ${ }^{1}$ and A. Zigler ${ }^{5}$ \\ ${ }^{1}$ Laboratori Nazionali di Frascati, Via Enrico Fermi 40, 00044 Frascati, Italy \\ ${ }^{2}$ INFN and Tor Vergata University, Via della Ricerca Scientifica 1, 00133 Rome, Italy \\ ${ }^{3}$ Sapienza University, Piazzale Aldo Moro 5, 00185 Rome, Italy \\ ${ }^{4}$ INFN Milano, via Celoria 16, 20133 Milan, Italy \\ ${ }^{5}$ Racah Institute of Physics, Hebrew University, 91904 Jerusalem, Israel
}

(Received 4 August 2017; accepted 10 October 2017; published online 30 October 2017)

\begin{abstract}
We report on the experimental characterization of the effect that a passive plasma lens in the overdense regime has on high-brightness bunch quality by means of $6 \mathrm{D}$ phase-space analysis. The passive lens is generated by confining hydrogen gas with a capillary tube pre-ionized with a highvoltage discharge. We observed that the optimum condition is retrieved at the end of the overdense regime with almost no effect on bunch brightness. The presence of gas jets, leaking from the hollow capillary end-points, extends the lens effects also outside of the capillary, resulting in longer focusing channels. Experimental results are supported with numerical simulations of the complete accelerator line together with the plasma channel section. Published by AIP Publishing.

https://doi.org/10.1063/1.4999010
\end{abstract}

Plasma-based accelerators (PBAs) $)^{1-3}$ hold great promise to accelerate charged beams to high energies in a compact way due to their capability to sustain accelerating gradients orders of magnitude larger than present radio frequency based technology. The recent developments in high-efficiency compact PBA are demonstrating the possibility to produce particle accelerators at a centimeter scale. ${ }^{4-6}$ Nowadays, most realizations of PBA make use of permanent magnet quadrupoles (PQMs) as optical elements for beam manipulation. However, most evident limitations of $\mathrm{PQMs}$ are the non-symmetric focusing, a small linearity area, emittance deterioration, and a scarce resilience against accidental irradiation. All those drawbacks could be overcome by envisioning a very compact PBA to be fully equipped with plasma based injection and extraction systems, for beam matching and transport, where focusing is demanded to plasma lens devices. A deep insight into the effect that plasma lenses have on high brightness beam quality is a necessary step to foreseen the flourishing of these new systems. Plasma focusing can occur for three different mechanisms: (i) by the radial fields of a largeamplitude plasma wave moving with the beam, ${ }^{7,8}$ (ii) by the azimuthal magnetic field produced by an axial current (active plasma lens), ${ }^{9-12}$ and (iii) by the self-focusing due to the shielding process produced when the background plasma reorganizes to conserve the overall neutrality (passive plasma lens). ${ }^{13-15}$ In this letter, we fully characterize, supporting our results with numerical simulations, the effects of a passive plasma lens on a high brightness electron beam. To date, no such detailed studies have been carried out, specifically in the case of capillary confined plasmas. We will put emphasis on unique behaviors clearly distinguishable in our measurements for the given setup: a long lasting overdense regime whose

a)alberto.marocchino@lnf.infn.it effective focusing depends only upon the mean bunch density, condition that may reduce synchronization constraints. The presence of plasma jets arises from the hollow capillary end points, resulting in an elongation of the plasma channel into the vacuum chamber. These jets extend passive lens effects outside the capillary and need to be taken into account. Finally, in the transition toward the underdense plasma lens regime, a setup is realized in which bunches can be focused with no brightness deterioration.

The experiment has been carried out at the SPARC_LAB test-facility. ${ }^{16}$ The SPARC_LAB photoinjector is used to produce a high quality electron bunch, 50 $\mathrm{pC}$ with $1 \mathrm{~mm}$-mrad normalized emittance and $0.2 \%$ energy spread. The bunch is accelerated by $2 \mathrm{~S}$-band RF sections to an energy of $127 \mathrm{MeV}$ and delivered at the capillary entrance with a transverse dimension of $\sigma_{x}=\sigma_{y}=95 \mu \mathrm{m}$ and a longitudinal rms-size of $\sigma_{z}=350 \mu \mathrm{m}$. The bunch number density is $n_{b}=6 \times 10^{12} \mathrm{~cm}^{-3}$. A capillary, in the VeroClear material (with negligible ablation, we have measured $79 \mu \mathrm{m}$ of ablation for 55000 shots), 3D-printed, $3 \mathrm{~cm}$ long, $1 \mathrm{~mm}$ internal diameter, is used to realize the plasma lens. It has been designed with two inlets, at $1 / 4$ and $3 / 4$ of the total length, for uniform gas filling. The two inlets are connected with a V-shaped pipe to a hydrogen generator that, through a valve, maintains a constant backing pressure of 300 mbar. ${ }^{17}$ At the edges of the capillary, we have inserted two copper electrodes, connected to a high-voltage generator $(20 \mathrm{kV})$ with a RC-time-constant of $\tau=R C \sim 300$ ns able to deliver $95 \mathrm{~A}$ in $900 \mathrm{~ns}$ (total duration). The discharge current is used to fully ionize the hydrogen gas, creating a uniform background plasma channel. Also, by changing the relative time between the discharge and the passage of the beam, we can operate at different plasma density configurations. The capillary, located in an interaction chamber, is aligned with the 

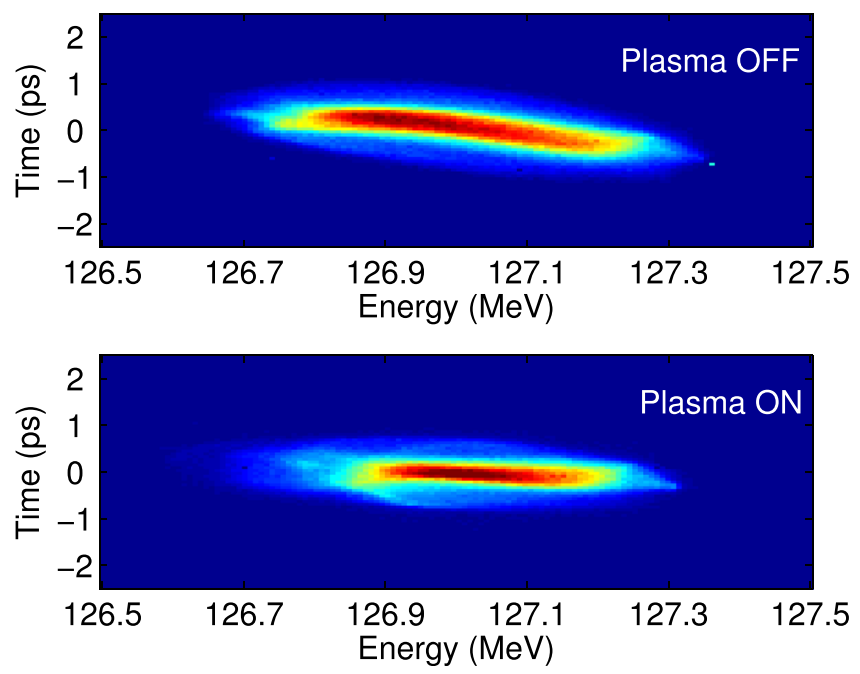

FIG. 1. Longitudinal phase space contour-plots. Top image: for reference, with no plasma and bottom image: at $-1.4 \mu \mathrm{s}$.

accelerator line and maintained in an ultrahigh-vacuum state $\left(9 \times 10^{-10} \mathrm{mbar}\right)$. The plasma density profile, inside and outside the capillary, is measured with the Stark broadening of the Balmer $\mathrm{H}_{\beta}$ line $(486.1 \mathrm{~nm})$. The electron density values are derived from the half-widths of the Balmer lines, acquired with a fast-gated intensified camera with a gate time of $100 \mathrm{~ns} .{ }^{18}$ To account for plasma fluctuations, every measurement results from averaging over 200 acquisitions. Characterization of the passive plasma lens effects, with specific attention to emittance and energy spread deterioration, requires a full $6 \mathrm{D}$ measurement. The bunch focusing has been measured by envelop reconstruction over three consecutive motorized and removable Ce:YAG screens placed at 20,37 , and $520 \mathrm{~cm}$ downstream the capillary. ${ }^{19,20}$ The last screen is also used for quadrupole scan rms-emittance $(100 \%$ charge) measurements, ${ }^{20,21}$ with a triplet placed between the second and the third YAG screen. Emittance measurements are performed taking into account the whole bunch since focusing is a whole bunch effect. Emittance deterioration is due to a general bunch phase space dilution, and experimental data supported by simulations help clarifying that emittance growth is a whole bunch effect and not only due to a limited bunch-edge particle effect. A RF-deflector ${ }^{22}$ is used to infer the bunch length, while a magnetic spectrometer allows energy measurements. The joint operation of these two latter techniques allows us to reconstruct the longitudinal phase-space (LPS).

In Fig. 1, we compare the LPS of a beam propagated in the neutral gas (top picture), i.e., without activating the discharge, and a beam coming from a plasma lens (bottom picture). Experimentally, the plasma has no influence on the beam energy, as expected from numerical simulations. The slight differences between the two plots are mainly due to RF instabilities and different quadrupole settings used to image the beam on the screen when the plasma is on or off. The energy spread is not affected by the plasma lens either, remaining at a constant $0.2 \%$ value. Overall energy spread fluctuation, together with the instrumental tolerance, is as small as $1 \%$, the value that is totally compatible with RF jitters.

The whole plasma lens experimental scan, which includes both the active and the passive lens regime, is reported in Figs. 2(a) and 2(b), together with the intensity of discharge current. The active plasma lens lasts for about $\sim 1.2 \mu \mathrm{s}$, from $-0.25 \mu \mathrm{s}$ to $0.8 \mu \mathrm{s}$, as reported in Fig. 2 . Times are relative to the peak discharge current. In the active plasma lens, at maximum focus, the focal spot is of $24 \mu \mathrm{m}$ with a jitter of $3 \mu \mathrm{m}$, and the fluctuation is about $12 \%$. The transition from active to passive lens is completed at $0.8 \mu \mathrm{s}$, and from $0.8 \mu \mathrm{s}$ up to $10 \mu \mathrm{s}$, the bunch experiences a passive lens focusing effect. To highlight the two different regimes, also at the graphical level, we have used color coding: Fig. 2 uses a light blue background color to mark the time window for the active plasma lens phase, while the passive lens has been marked with a light yellow background. In this long lasting passive lens effect, we can further distinguish an overdense regime $\left(n_{b} / n_{p} \ll 1\right.$, with $n_{p}$ the plasma

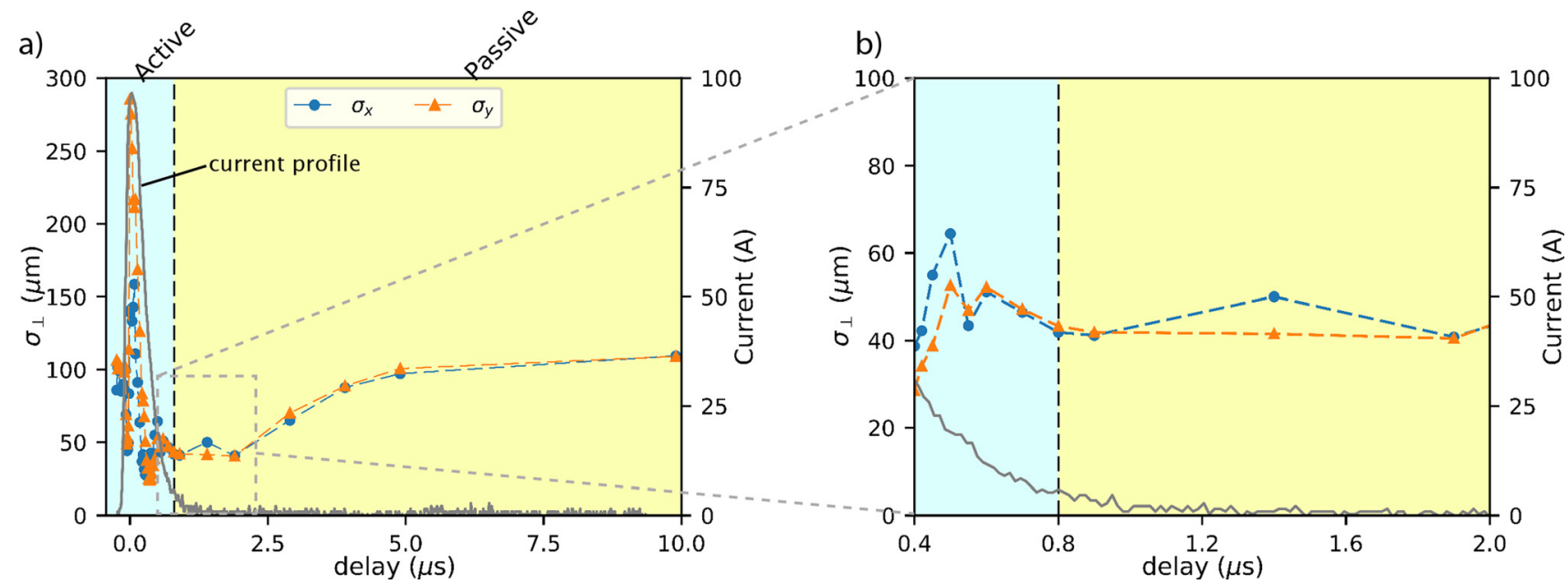

FIG. 2. Measurements of the transverse bunch rms-dimension as a function of the current delay time. Blue circles correspond to $\sigma_{x}$, dark yellow triangles to $\sigma_{y}$, while the current profile is overimposed in gray color. Sub-figure (a) plots the entire experimental scan, and subsection (b) focuses on the delay of $0.4-2.0 \mu$ s, the early phase of the active lens, characterized by a constant focusing gradient. Rms-dimensions were measured at the first screen, $17.5 \mathrm{~cm}$ from the capillary center. Background colors have been used to identify the Active lens, light blue, and Passive lens, light yellow. Subplot (b) is a zoom-in image for the timeinterval $(0.4 \mu \mathrm{s}, 2 \mu \mathrm{s})$. 
background number density) acting in the time window from $0.8 \mu \mathrm{s}$ to approximately $2 \mu \mathrm{s}$, while the remaining time corresponds to an underdense regime $\left(n_{b} / n_{p} \gg 1\right)$. From $0.8 \mu \mathrm{s}$ on, the current is too low to produce any active focusing, and the self-focusing gradient dominates due to the overdense regime. From Fig. 2(b), we deduce that the current at the beginning of the overdense regime is approximately $5 \mathrm{~A}$, corresponding to a gradient $d B / d r=4 \mathrm{~T} / \mathrm{m}$, while the net self-focusing gradient ${ }^{15}$ is evaluated to be $2 \pi e^{2} n_{b} \sim 30 \mathrm{~T} / \mathrm{m}$. For time larger than $1.2 \mu \mathrm{s}$, the current completely loses the organized avalanche behavior and the background electrons have a random thermal motion. We notice also that the most relevant passive lens phase spans from $0.8 \mu$ s to $2 \mu$ s. This phase is characterized by a relatively short and constant focal length lasting for more than a $\mu$ s. Its long duration makes this phase suitable for applications, e.g., single or multi-bunch plasma based accelerator, since it significantly reduces synchronization constraints. Active plasma lenses do not offer the same flexibility because the bunch passage through the capillary requires a strict synchronization with the desired current value.

Stark-broadening measurements of the plasma density, both inside and outside the capillary, are reported in Fig. 3. The measured values have been acquired off-line by overimposing three distinct sets of measures, the two jets and the central plasma. The plasma temperature inside and outside the capillary is inferred to be around $2-3 \mathrm{eV}$. Figure 3 shows particularly elongated jets with a fairly constant density, in space, for the duration of the early passive lens phase. The jet shape is qualitatively diagnosed by collecting the self-

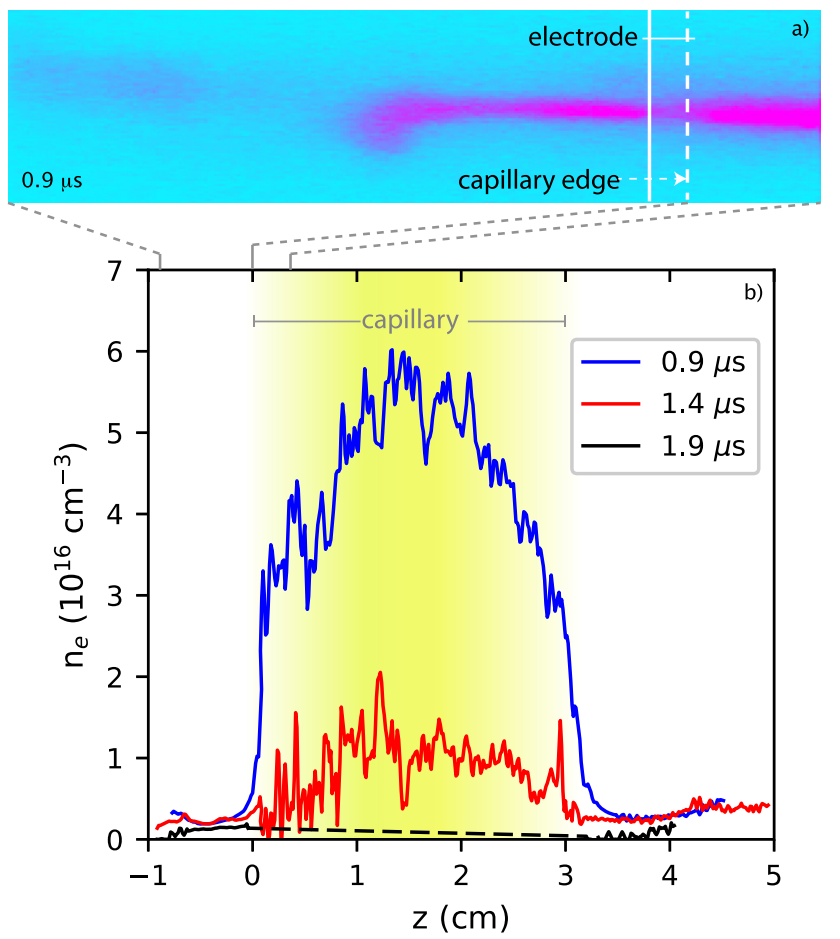

FIG. 3. (a) Jet shape at $0.9 \mu$ s diagnosed by imaging the self-emitted light onto an intensified gated camera, capillary side where the bunch enters. (b) Stark-broadening measurements of the electron plasma number density, inside and outside the capillary, for three times, $0.9,1.4$, and $1.9 \mu \mathrm{s}$. At the latest time, the density within the capillary has been partially reconstructed (dashed line). emitted light with an intensified gated camera, and Fig. 3(a) reports the $0.9 \mu$ s case arising from the capillary edge on the bunch impinging side (photo-cathode side). The jets are nonsymmetric, extending for about $1 \mathrm{~cm}$ on the side where the bunch impinges and for almost $2 \mathrm{~cm}$ on the other side, and from times between 0.9 and $1.4 \mu \mathrm{s}$, they have a density of approximately $1.2 \times 10^{15} \mathrm{~cm}^{-3}$. Those plasma ejecta occur supersonically with a Mach number of $\mathrm{Ma} \sim 2$, showing some degree of collimation. Jets may have a deep impact on beam dynamics, depending on their extension and on their density: the critical parameter is the product between the bunch length and plasma wavenumber $k_{p} \sigma_{z}$. In order to evaluate their effects, we performed beam dynamics simulations in the plasma by employing the code Architect. ${ }^{23-25}$ Since the scope is to highlight the safe focusing regions, with little impact on emittance, we employed a simplified setting consisting of a $5 \mathrm{~mm}$ long plasma with longitudinally constant density. Final beam emittance values are reported in Fig. 4 for different plasma density values. A resonance condition occurs when the bunch length is comparable to the inverse plasma wavenumber $k_{p} \sigma_{z} \sim 1$, while transport with reduced emittance dilution is found when the background plasma $n_{p}$ is either very dense $\left(k_{p} \sigma_{z} \gg 1\right)$ or very sparse $\left(k_{p} \sigma_{z} \ll 1\right)$. The maximum emittance degradation can be explained as follows: The electric discharge ionizes the hydrogen gas keeping it globally and locally neutral; neutrality is perturbed when an electron bunch is injected into the plasma. To conserve neutrality, plasma electrons screen the bunch by selfre-organizing their structure and producing an ion bubble surrounding the charged bunch. The background electron ejection is due to coulomb repulsion by bunch electrons $\left[F_{\perp}=-e\left(E_{r}-v_{z} B_{\theta}\right)\right]$. The transverse momentum gained by the plasma electrons is proportional to the charge and length of the beam. In a fully blowout regime, the large bunch charge immediately ejects background electrons completely, soaking the entire bunch into an ion bubble. When charges are modest, as in our case, plasma electrons have to travel some distance within the bunch before being diverted. As a consequence, the bunch head is immersed in a uniform neutral plasma, while the tail sits in the ion bubble. The head and tail then experience two different electric fields. While

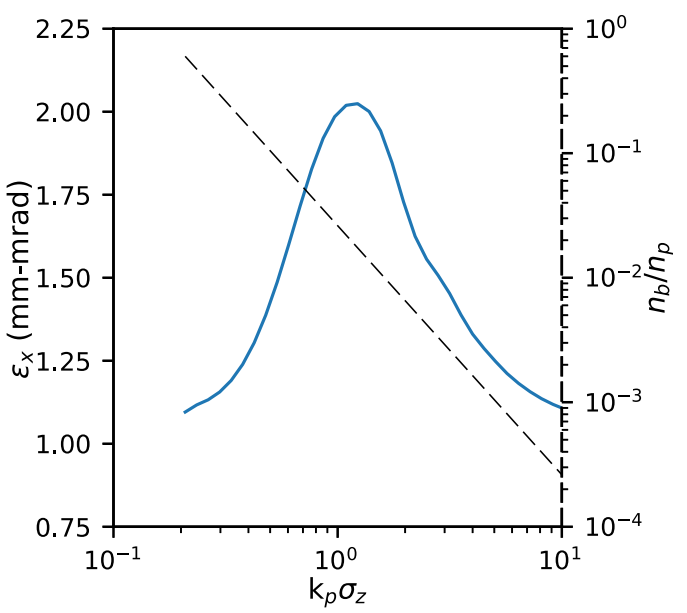

FIG. 4. Emittance growth as a function of the product plasma number density and bunch length. This image is used to identify the resonance behavior around $k_{p} \sigma_{z}=1$. Simulations use the simulated start-to-end bunch. 
the head is not confined by any transverse force, the tail is transversally focused by the bubble fields. This double behavior along the length of the same bunch causes a quality deterioration by increasing emittance. From Fig. 4, we deduce that the ideal focusing is found either at high densities or at low densities. Despite that these two conditions are mathematically equivalent, they correspond to (very) different laboratory scenarios. The former is found throughout the transition from active to passive lens, when high density characterizes the areas both inside and outside the capillary. The latter, instead, realizes a fully passive lens regime with a more uniform density profile just above $n_{p} \sim 3 \times 10^{13} \mathrm{~cm}^{-3}$.

For experimental data interpretation, simulations have been performed by using in cascade the tracking code $\mathrm{GPT}^{26}$ with Architect. GPT is initially used to track the bunch particles from the photo-cathode up to the plasma. The tracked bunch is then imported into Architect to simulate the evolution within the plasma. At the end of the plasma channel, particles are imported back, once more, into GPT to track them up to the diagnostic screens, for a final direct comparison with experimental results. To this end, the background plasma profile used in the Architect code is obtained by remapping the experimental data of Fig. 3, assuming no radial modulations since at the density of interest, small radial perturbations or radial density fading has a negligible effect on the bunch quality and dynamics. Simulations are performed with a million particles for the plasma section, where cylindrical symmetry has been assumed. A longitudinal and transverse resolution of $5 \mu \mathrm{m}$ has been used with a moving window technique, $3 \mathrm{~mm}$ wide, and a 5.3 fs integration timestep. The reconstructed emittance via computer simulations is compared with experimental measurements in Fig. 5. We find a very good agreement between the two sets of data. Once delivered to the plasma, electron bunches travel through a continuous gradient of density values which causes a constant and a non-uniform emittance growth. Significantly simplifying, we notice that the growth occurs at

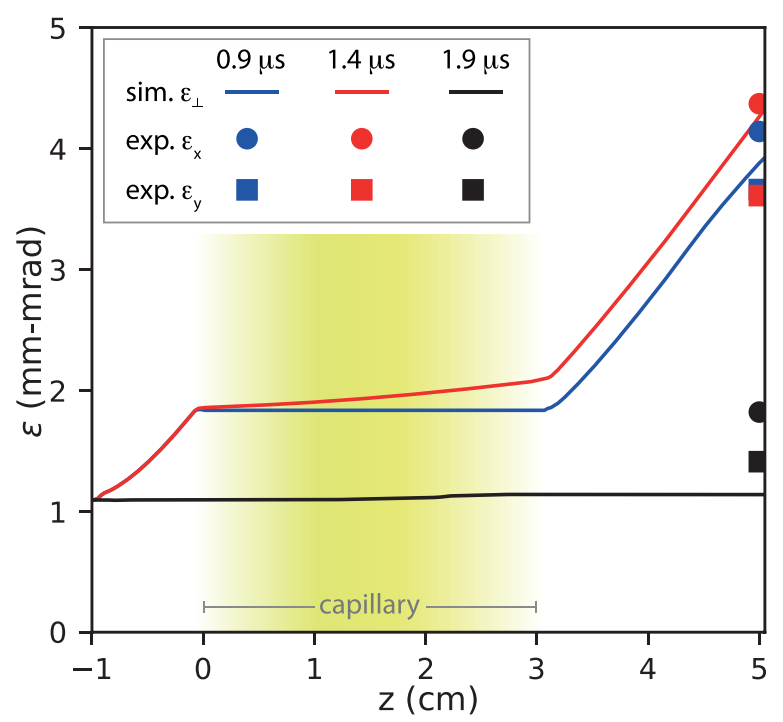

FIG. 5. Plot of the normalized transverse rms-emittance as a function of $z$. The numerically computed emittance is plotted with a solid line, and the experimental values are reported on the right edge. The $\varepsilon_{x}$ experimentally measured quantities are reported with a circle and $\varepsilon_{y}$ with a square. three different paces due to the different density found in the plasma jets, compared to the density within the capillary. With a delay of $1.9 \mu \mathrm{s}$, the plasma has flattened out and finally moved away from the emittance resonance condition, keeping it at the injection level. Envisioning the use of compressed bunches in the same experimental scenario, i.e., with the same capillary and plasma density evolution, we will always satisfy an overdense regime in the limit of $k_{p} \sigma_{z} \ll 1$, ensuring a strong symmetric focusing together with brightness preservation.

In this paper, we have presented the experimental characterization of the passive lens effect on high brightness bunches. The passive lens effect begins when the electron avalanche behavior (active phase) produced by the current discharge comes to an end. In our setups, active lenses are characterised by stronger focusing gradients, and however, the focusing is subject to aberration effects if the bunch hits the region toward the capillary edge where the magnetic field nonlinear bends or by other nonlinear aberrations (e.g., coma) if the bunch is not injected at the capillary centre where the poloidal B field crosses the zero point. Passive lenses overcome these limits since the focusing is operated by a bath of electrons characterised by thermal motion with no geometrical centre. The uniform background (with no geometrical centre) allows the bunch to be injected and focused for any injection radius. The passive lens offers a gentler focusing compared to the active lens, still of interest to be used in a conventional RF line, and in the transition from the overdense to the underdense regime, the focusing occurs with no phase-space deterioration, making this technique appealing.

This work was partially supported by the EU Commission in the Seventh Framework Program, Grant Agreement No. 312453-EuCARD-2, the Italian Research Minister FIRB framework, Project No. RBFR12NK5K, and the European Union Horizon 2020 research and innovation programme under Grant Agreement No. 653782 (EuPRAXIA).

\footnotetext{
${ }^{1}$ T. Tajima and J. M. Dawson, Phys. Rev. Lett. 43, 267 (1979).

${ }^{2}$ P. Chen, J. Dawson, R. W. Huff, and T. Katsouleas, Phys. Rev. Lett. 54, 693 (1985).

${ }^{3}$ M. Litos, E. Adli, W. An, C. Clarke, C. Clayton, S. Corde, J. Delahaye, R. England, A. Fisher, J. Frederico et al., Nature 515, 92 (2014).

${ }^{4}$ I. Blumenfeld, C. E. Clayton, F.-J. Decker, M. J. Hogan, C. Huang, R. Ischebeck, R. Iverson, C. Joshi, T. Katsouleas, N. Kirby, W. Lu, K. A. Marsh, W. B. Mori, P. Muggli, E. Oz, R. H. Siemann, D. Walz, and M. Zhou, Nature 445, 741 (2007).

${ }^{5}$ B. M. Hegelich, B. Albright, J. Cobble, K. Flippo, S. Letzring, M. Paffett, H. Ruhl, J. Schreiber, R. Schulze, and J. Fernández, Nature 439, 441 (2006).

${ }^{6}$ E. Kallos, T. Katsouleas, W. D. Kimura, K. Kusche, P. Muggli, I. Pavlishin, I. Pogorelsky, D. Stolyarov, and V. Yakimenko, Phys. Rev. Lett. 100, 074802 (2008).

${ }^{7}$ J. Rosenzweig, B. Breizman, T. Katsouleas, and J. Su, Phys. Rev. A 44, R6189 (1991).

${ }^{8}$ R. Fedele, U. de Angelis, and T. Katsouleas, Phys. Rev. A 33, 4412 (1986).

${ }^{9}$ W. K. H. Panofsky and W. Baker, Rev. Sci. Instrum. 21, 445 (1950).

${ }^{10}$ E. Boggasch, A. Tauschwitz, H. Wahl, K.-G. Dietrich, D. Hoffmann, W. Laux, M. Stetter, and R. Tkotz, Appl. Phys. Lett. 60, 2475 (1992).

${ }^{11}$ J. Van Tilborg, S. Steinke, C. Geddes, N. Matlis, B. Shaw, A. Gonsalves, J. Huijts, K. Nakamura, J. Daniels, C. Schroeder et al., Phys. Rev. Lett. 115, 184802 (2015).
} 
${ }^{12}$ R. Pompili, M. Anania, M. Bellaveglia, A. Biagioni, F. Bisesto, E. Chiadroni, A. Cianchi, M. Croia, A. Curcio, D. D. Giovenale et al., Nucl. Instrum. Methods Phys. Res., Sect. A 829, 17-23 (2016).

${ }^{13}$ P. Chen, K. Oide, A. Sessler, and S. Yu, Phys. Rev. Lett. 64, 1231 (1990).

${ }^{14}$ H. Nakanishi, Y. Yoshida, T. Ueda, T. Kozawa, H. Shibata, K. Nakajima, T. Kurihara, N. Yugami, Y. Nishida, T. Kobayashi et al., Phys. Rev. Lett. 66, 1870 (1991).

${ }^{15}$ J. Su, T. Katsouleas, J. Dawson, and R. Fedele, Phys. Rev. A 41, 3321 (1990).

${ }^{16}$ M. Ferrario, D. Alesini, M. Anania, A. Bacci, M. Bellaveglia, O. Bogdanov, R. Boni, M. Castellano, E. Chiadroni, A. Cianchi et al., Nucl. Instrum. Methods B 309, 183 (2013).

${ }^{17}$ A. Biagioni, M. Anania, M. Bellaveglia, E. Chiadroni, A. Cianchi, D. D. Giovenale, G. D. Pirro, M. Ferrario, F. Filippi, A. Mostacci et al., J. Instrumentation 11, C08003 (2016).

${ }^{18}$ F. Filippi, M. Anania, A. Biagioni, E. Chiadroni, A. Cianchi, M. Ferrario, A. Mostacci, L. Palumbo, and A. Zigler, J. Instrumentation 11, C09015 (2016).

${ }^{19}$ R. Pompili, M. P. Anania, M. Bellaveglia, A. Biagioni, G. Castorina, E. Chiadroni, A. Cianchi, M. Croia, D. D. Giovenale, M. Ferrario, F.
Filippi, A. Gallo, G. Gatti, F. Giorgianni, A. Giribono, W. Li, S. Lupi, A. Mostacci, M. Petrarca, L. Piersanti, G. D. Pirro, S. Romeo, J. Scifo, V. Shpakov, C. Vaccarezza, and F. Villa, New J. Phys. 18, 083033 (2016).

${ }^{20}$ A. Cianchi, D. Alesini, M. Anania, A. Bacci, M. Bellaveglia, M. Castellano, E. Chiadroni, D. Di Giovenale, G. Di Pirro, M. Ferrario et al., Phys. Rev. Spec. Top. Accel. Beams 18, 082804 (2015).

${ }^{21}$ A. Mostacci, M. Bellaveglia, E. Chiadroni, A. Cianchi, M. Ferrario, D. Filippetto, G. Gatti, and C. Ronsivalle, Phys. Rev. Spec. Top. Accel. Beams 15, 082802 (2012).

${ }^{22}$ D. Alesini, G. Di Pirro, L. Ficcadenti, A. Mostacci, L. Palumbo, J. Rosenzweig, and C. Vaccarezza, Nucl. Instrum. Methods Phys. Res. Sect. A 568, 488 (2006).

${ }^{23}$ A. Marocchino, F. Massimo, A. Rossi, E. Chiadroni, and M. Ferrario, Nucl. Instrum. Methods Phys. Res., Sect. A 829, 386-391 (2016).

${ }^{24}$ A. Marocchino and F. Massimo, Architect: First Release (Zenodo-CERN, 2016).

${ }^{25}$ F. Massimo, S. Atzeni, and A. Marocchino, J. Comput. Phys. 327, 841 (2016).

${ }^{26}$ M. D. Loos and S. Van der Geer, in 5th European Particle Accelerator Conference (1996), p. 1241. 